\title{
The Big Bang Observer: High Laser Power for Gravitational Wave Astrophysics
}

\author{
Gregory M Harry ${ }^{1}$, William Folkner ${ }^{2}$, Peter Fritschel ${ }^{1}$, E Sterl Phinney ${ }^{3}$, Daniel A Shaddock ${ }^{2}$ \\ ${ }^{1}$ LIGO Laboratory, Massachusetts Institute of Technology, \\ Cambridge MA, 02139; ph 617-258-8279, fax 617-253-7014, \\ gharry@ligo.mit.edi; ${ }^{2}$ Jet Propulsion Laboratory, California Institute of Technology, \\ Pasadena CA, 91109; ${ }^{3}$ California Institute of Technology, Pasadena CA, 91125
}

\begin{abstract}
The Big Bang Observer(BBO) is a proposed space-based gravitational wave detector which will look for the stochastic background of gravitational waves from the early universe. The positions of three free test masses, $50,000 \mathrm{~km}$ apart, will be monitored by laser interferometry. To reduce noise, $300 \mathrm{~W}$ of $355 \mathrm{~nm}$ light obtained from frequency-tripling the output of Nd:YAG lasers will be used. These lasers and other technology necessary for BBO will require significant research before a potential launch date of 2025 .
\end{abstract}

OCIS Codes 350.1270, 140.5680

The Big Bang Observer (BBO) is a proposed follow-on mission to the Laser Interferometer Space Antenna (LISA) which is designed to detect the stochastic background of gravitational waves from the early universe. It will provide sensitivity between the LISA [1] band (from roughly $1 \mathrm{mHz}-1 \mathrm{~Hz}$ ) and the ground-based Advanced LIGO [2] (Laser Interferometer Gravitational-wave Observatory) (from roughly 10-3000 Hz). This bandwidth will be obtained by having the spacecraft 50,000 kilometers apart compared to the $5 \times 10^{6} \mathrm{~km}$ planned for LISA. More details about BBO can be found in recent papers and reports [3-5].

Shot noise will limit the sensitivity in BBO's most sensitive band, according to [1]

$$
S_{x}(f)=h c \lambda^{3} L^{2} /\left(2 \pi^{2} \eta P D^{4}\right),
$$

where $S_{x}$ is the position noise spectral density, $\lambda$ is the wavelength of light, $L$ is the spacecraft separation, $\eta$ is the loss in the optics, $P$ is the laser power, and $D$ is the collection mirror diameter. To lower this noise, each spacecraft will have two $300 \mathrm{~W}$ beams operating at $355 \mathrm{~nm}$, obtained from frequency tripling $1.064 \mathrm{~nm}$ light from Nd:YAG lasers. These lasers will be projected between the three spacecraft to monitor their relative positions, and hence any disturbances in space-time from gravitational waves.

After traversing $5 \times 10^{7} \mathrm{~m}$, the light will be collected by $2.5 \mathrm{~m}$ diameter mirrors. After diffraction losses, the incoming beam will be about $8 \mathrm{~W}$. The incoming plane wave will become an Airy disk upon reflection from the mirror. This beam will be interfered with $8 \mathrm{~W}$ of the Gaussian-profile beam local to the spacecraft. A strategy to keep down the resulting contrast defect from interfering two differently shaped beams will be necessary. The position of one of the test masses will then be controlled by feedback to keep the interfering beams on a dark fringe. This will help keep down the power handling requirements of the photodiodes. With such high power beams and high contrast defect, it may be necessary to have multiple photodiodes to handle the power of even the "dark" fringe.

One of the inter-spacecraft lengths will serve as a reference cavity, to control the frequency of one of the lasers. The other lasers will then be phase locked to this master laser. Each laser will pass through a local Fabry-Perot cavity on each spacecraft, which will serve as both a passive mode cleaner to stabilize beam direction and as a frequency reference. This cavity will have a finesse of about 100 to provide the necessary filtering and provide low enough shot noise in the frequency control loop. Each beam will have RF phase modulation at about $10 \mathrm{MHz}$ for signal detection. This will allow the signal to be above low frequency technical noises. The question arises of where the $\mathrm{RF}$ phase modulation is applied, before or after this cavity. If after, the full $300 \mathrm{~W}$ will have to be passed through an electro-optic modulator. If before, the modulation would have to go through the Fabry-Perot cavity, which would preclude any intensity noise reduction to occur in the cavity and thus require the laser to be shot noise limited when generated. Either of these strategies would require significant research to utilize.

[1] P. L. Bender, et al., "LISA Pre-Phase A Report; Second Edition", MPQ 233 (1998).

[2] P. Fritschel, Second generation instruments for the laser interferometer gravitational wave observatory," in GravitationalWave Detection, M. Cruise and P. Saulson, Proc. SPIE 4856, pp. 282291, (2003).

[3] E. S. Phinney et al., "The Big Bang Observer: Direct detection of gravitational waves from the birth of the Universe to the Present," NASA Mission Concept Study (2004).

[4] J. Crowder, N. J. Cornish, Phys. Rev. D 72, 083005 (2005).

[5] G. M. Harry, P. Fritschel, D. A. Shaddock, W. Folkner, and E. S. Phinney Class. Quantum Grav. 23 4887 (2006); Class. Quantum Grav. 237631 (2006). 